\title{
ENHANCED EXCITON-PHONON INTERACTION IN STRAINED ZnCdSe/ZnSe QUANTUM WELL STRUCTURES
}

\author{
M. GoDlewsKi \\ Institute of Physics, Polish Academy of Sciences \\ Al. Lotników 32/46, 02-668 Warsaw, Poland \\ K. LEONARDI AND D. HOMMEL
}

Institute of Solid State Physics, Bremen University, 28334 Bremen, Germany

\begin{abstract}
Radiative recombination processes in pseudomorphic $\mathrm{ZnCdSe} / \mathrm{ZnSe}$ structures are compared to those observed in strain-relaxed structures grown on GaAs substrates with thick $\mathrm{ZnSe}$ bufter layers. From the temperature dependence of the photoluminescence line width we evaluate the strength of exciton-phonon interaction with acoustic (dominant at lower temperatures) and optical phonons. Stronger exciton-phonon interaction is observed for pseudomorphic structures. Such enhanced exciton-phonon interaction is likely responsible for a faster photoluminescence deactivation at increased temperatures. We also report different exciton properties (photoluminescence intensity, width, strength of exciton-phonon interaction) in quantum well of a given width but in structures grown with different order of quantum wells. More stable photoluminescence (with increasing temperature) is observed for a given quantum well if it is closer to the buffer layer and not to the cap layer.
\end{abstract}

PACS numbers: 68.60.-p, 76.70.Hb

\section{Exciton-phonon interaction in $2 \mathrm{D}$ systems}

In this work we discuss exciton-phonon interaction in $\mathrm{ZnCdSe} / \mathrm{ZnSe}$ heterostructures, which were either pseudomorphic (strained) or had a thick $\mathrm{ZnSe}$ buffer layer on top of a GaAs substrate, resulting in a strain relaxation. We report quite different exciton-phonon interaction in these two types of structures.

There are two types of the exciton-phonon interactions - interaction with acoustic phonons and interaction with optical phonons. In the low temperature limit the former interaction dominates [1]. This interaction is described by either a deformation potential (stronger interaction) or a piezoelectric interaction (weaker [2]). In 2D systems exciton-phonon interaction becomes two orders in 
magnitude stronger than that present in bulk samples [2]. This is due to the arbitrary values of $k_{z}$ ( $z$ is normal to a quantum well (QW) plane) of phonons, which may interact with excitons in a $\mathrm{QW}$ plane. Ilowever, the strength of the interaction depends critically on an extent of exciton localization. The exciton-phonon interaction becomes weaker for localized excitons [2]. This relates to the fact that the magnitude of wave vectors of phonons interacting with localized excitons is limited to within few times of the localization length. For free excitons in $10 \mathrm{~nm}$ wide $\mathrm{QW}$ in GaAs/AlGaAs heterostructure the theory predicts $\gamma_{\mathrm{ph}} \approx 34 \mu \mathrm{eV} / \mathrm{K}$ [3]. However, due to exciton localization present at low temperature, the experimentally measured interaction constants are usually few times smaller $[4,5]$. Also the character of the interaction is different for the localized excitons. Here excitons can migrate/tunnel among localized states due to phonon-assisted processes. Such interaction determines a homogeneous line width of photoluminescence (PL) emission and PL intensity of localized excitons. Due to phonon-assisted tunneling transitions of localized excitons their PL line width $(\Delta(T))$ can be described in a similar manner as that for mobile excitons: $\Delta(T)=\Delta_{0}+\gamma_{\mathrm{ph}} T$, where $\Delta_{0}$ is $0 \mathrm{~K}$ PL line width and $\gamma_{\mathrm{ph}}$ is exciton-phonon (acoustic phonons) interaction constant.

\section{Samples}

A series of four $\mathrm{ZnCdSe} / \mathrm{ZnSe}$ heterostructures grown by $\mathrm{MBE}$ on (001) GaAs substrate was studied. Each of these structures contained two relatively thick $\mathrm{ZnCdSe}(12 \%$ of $\mathrm{Cd}$ ) QWs (14 and $8 \mathrm{~nm}$ wide) separated by $35 \mathrm{~nm}$ thick $\mathrm{ZnSe}$ barriers with $17 \mathrm{~nm}$ wide ZnSe cap layer. They differ by order of QWs. The structure with the $14 \mathrm{~nm}$ wide QW being closer to the GaAs/ZnSe interface will be called "normal" and the one with the opposite order of QWs will be called "inverted" structure. Two structures (one normal, one inverted) were pseudomorphic. These structures were grown with a relatively thin $\mathrm{ZnSe}$ buffer layer $(35 \mathrm{~nm}$ wide) on top of a GaAs substrate. Two other structures were grown on a thick $\mathrm{ZnSe}$ buffer layer (1000 $\mathrm{nm}$ wide) and were strain-relaxed structures.

\section{Photoluminescence studies}

Photoluminescence, PL excitation (PLE) and temperature dependence of the emission were measured for all heterostructures studied. For the inverted pseudomorphic structure the PL is dominated by the donor bound exciton (DBE) emission from the $8 \mathrm{~nm}$ wide QW. By selecting the PLE encrgy we could enhance the contribution of either free (FE) or DBE transitions. Temperature dependence of PL emission (intensity, position and width - full width at half maximum (FWHM)) was measured. Up to $60 \mathrm{~K}$ both the intensity and the spectral position of the PL bands were weakly temperature dependent. For $T>100 \mathrm{~K} \mathrm{PL}$ emissions shift down in the energy and their intensity decreases fast. The FWHM of PLs increases fast with an increasing temperature, as can be seen in Fig. 1 for the $8 \mathrm{~nm}$ QW PL. For this QW the relative contribution of the FE and DBE PLs was difficult to be resolved and the low temperature results are disturbed by the contribution of the DBE transitions. Ilowever, we found that $\gamma_{\mathrm{ph}}$ interaction constants are very similar for the two types of the excitonic transitions. Up to 


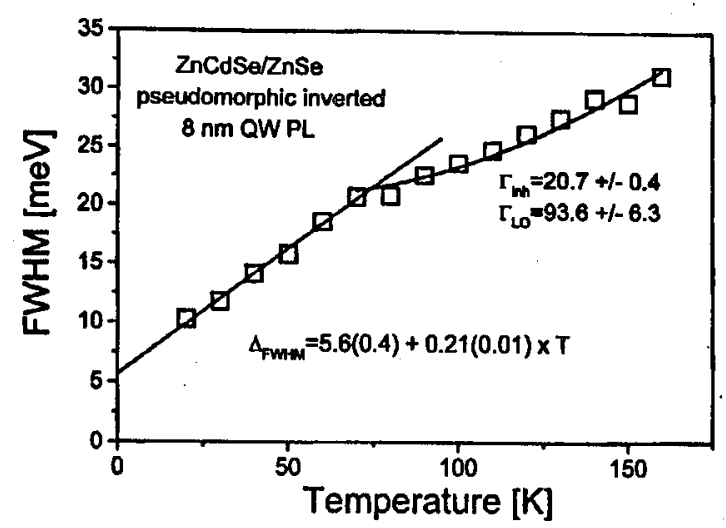

Fig. 1. Temperature dependence of the PL line width (FWIIM) for the $8 \mathrm{~nm} \mathrm{QW}$ emission from the inverted pseudomorphic structure.

about $70 \mathrm{~K}$ interaction with acoustic phonons dominates, which results in a linear increase in the PL line width described by an interaction constant of about $\gamma_{\mathrm{ph}} \approx 0.21 \mathrm{meV} / \mathrm{K}$. For higher temperatures interaction with optical phonons becomes important $\left(\Gamma_{\mathrm{LO}} \approx 94 \mathrm{meV} / \mathrm{K}\right)$. This results in an exponential increase in the PL line width at increased temperatures, as expected for the interaction with optical phonons [6]. The value of $94 \mathrm{meV} / \mathrm{K}$ is noticeably larger than $60 \mathrm{meV} / \mathrm{K}$ for bulk $\mathrm{ZnSe}[6]$.

Depending on the excitation conditions PL of the normal pseudomorphic structure was dominated by either $8 \mathrm{~nm}$ or $14 \mathrm{~nm} \mathrm{QW}$ emissions. Also the relative intensity of the FE and the DBE transitions could be changed. A fast decrease in the PL intensity was observed with an increasing temperature. This process could not, however, be described by a single exponential decrease. Also the shift of the PL position could not be described by a definite dependence. The temperature dependencies of the PL line width for FE and DBE emissions from the $8 \mathrm{~nm}$ QW and the $14 \mathrm{~nm} \mathrm{QW}$ were measured. IIere the strongest interaction with acoustic phonons is observed for the DBE $8 \mathrm{~nm}$ QW emission $\left(\gamma_{\mathrm{ph}} \approx 190 \mu \mathrm{eV} / \mathrm{K}\right)$. For the $8 \mathrm{~nm}$ QW excitons are strongly localized and only a weak interaction with acoustic phonons is observed. In the case of the $14 \mathrm{~nm}$ QW both PLs have very similar interaction constants with acoustic phonons, as we also observed for the inverted structure. PL intensity of the normal pseudomorphic structure decreases too fast with an increasing temperature to observe any evidence of interaction with optical phonons.

In the PL spectrum for the inverted strain-relaxed structure only a weak contribution of DBE PLs was observed. Down in the energy shift of the PL spectral positions with an increasing temperature was observed. This shift is slightly slower than that observed for the pseudomorphic structures. It also starts at lower temperature $(50 \mathrm{~K}$ vs. $100 \mathrm{~K}$ for the pseudomorphic structures). The PL intensity is deactivated with the energy nearly twice larger than that observed for the strained structures. This means that PL in strain-relaxed structures is more stable and can 
be observed at higher temperatures. In turn, interaction with acoustic phonons is here weaker. For the FE emission in the $8 \mathrm{~nm}$ wide QW this interaction is nearly three times weaker than that observed for excitons in the $8 \mathrm{~nm}$ wide QW in the pseudomorphic structures. For the $14 \mathrm{~nm}$ QW excitons were localized up to $50 \mathrm{~K}$. For higher temperature PL line starts to broaden, but we could not determine accurately the relevant constant of exciton-phonon interaction for this QW.

The PL spectrum observed for the normal strain-relaxed structure was dominated by the FE PL from the $14 \mathrm{~nm}$ wide QW. The relative intensity of the FE and DBE PLs could be changed by measuring the PL at different excitation conditions. Temperature dependencies of the PL intensity and of the PL spectral position were quite similar to those measured for the inverted structure. Temperature dependencies of the FWHM of FE PLs in the 8 and $14 \mathrm{~nm}$ wide QWs were measured. For the $14 \mathrm{~nm} \mathrm{QW}$ excitons are strongly localized up to $50 \mathrm{~K}$ (temperature independent line width) and then start to tunnel among various sites in a $\mathrm{QW}$ plane (phonon-assisted process). The localized character of excitons in the $14 \mathrm{~nm}$ QW is also reflected by a smaller exciton-phonon interaction constant, which is about three times smaller from that for excitons in the $8 \mathrm{~nm}$ wide QW. Interaction with acoustic phonons dominates up to $100 \mathrm{~K}$. The interaction constants with optical phonons, given in Tables I and II, were determined from a relatively small temperature region and thus are of a limited accuracy.

TABLE I

Exciton-phonon interaction for the $8 \mathrm{~nm}$ wide QWs in $\mathrm{ZnCdSe} / \mathrm{ZnSe}$ structures.

\begin{tabular}{c|c|c|c|c}
\hline \hline 8 nm QW & FE PL & FE PL & DBE PL & DBE PL \\
& $\gamma_{\mathrm{ph}}[\mu \mathrm{eV} / \mathrm{K}]$ & $\Gamma_{\mathrm{LO}}[\mu \mathrm{eV} / \mathrm{K}]$ & $\gamma_{\mathrm{ph}}[\mu \mathrm{eV} / \mathrm{K}]$ & $\Gamma_{\mathrm{L} O}[\mu \mathrm{eV} / \mathrm{K}]$ \\
\hline pseudomorphic inverted & 210 & 94 & 210 & - \\
pseudomorphic normal & - & - & 190 & - \\
strain-relaxed inverted & 86 & 110 & - & - \\
strain-relaxed normal & 136 & $43 ?$ & - & -
\end{tabular}

TABLE II

Exciton-phonon interaction for the $14 \mathrm{~nm}$ wide QWs in $\mathrm{ZnCdSe} / \mathrm{ZnSe}$ structures.

\begin{tabular}{c|c|c|c|c}
\hline \hline $14 \mathrm{~nm} \mathrm{QW}$ & FE PL & FE PL & DBE PL & DBE PL \\
& $\gamma_{\mathrm{ph}}[\mu \mathrm{eV} / \mathrm{K}]$ & $\Gamma_{\mathrm{LO}}[\mu \mathrm{eV} / \mathrm{K}]$ & $\gamma_{\mathrm{ph}}[\mu \mathrm{eV} / \mathrm{K}]$ & $\Gamma_{\mathrm{LO}}[\mu \mathrm{eV}]$ \\
\hline pseudomorphic inverted & - & - & - & - \\
pseudomorphic normal & 95 & - & 95 & - \\
strain-relaxed inverted & - & $\approx 25$ & - & - \\
strain-relaxed normal & 56 & $11 ?$ & - & -
\end{tabular}




\section{Discussion}

The present study shows quite different exciton properties in strained and strain-relaxed structures. In the latter case, the PL is much more stable and can be observed up to the room temperature. PL emissions from the pseudomorphic structures are slightly shifted up in the energy and show different temperature dependencies of their spectral positions and intensity. This is another consequence of the strain. Another interesting observation of the present study is that the PL properties (e.g. the temperature stability) depend on the QWs order. In some cases we observed quite different PL intensities for two structures differing only by a QW order. It remains to be checked if such dependence relates to the fact that a given $\mathrm{QW}$ is closer to the GaAs/ZnSe interface or to the cap layer.

In Tables I and II we summarize the constants of exciton-phonon interaction determined in the present investigations. As mentioned in the introduction, this interaction depends on an extent of exciton localization, i.e., on a morphology of interfaces. Moreover, for the optical phonons it depends strongly on a $\mathrm{QW}$ width [7]. Even though we could not determine the interaction constants for all the emissions studied, Tables I and II clearly show differences in their values for QWs of the same width in the strained and in the strain-relaxed structures. In the latter case smaller interaction constants are observed reflecting more localized character of excitons in such structures. It is interesting to notice that stronger exciton-phonon interaction observed for the strained structures is accompanied by a faster decrease in the PL intensity at increased temperatures.

\section{References}

[1] D.S. Citrin, Phys. Rev. B 47, 3832 (1993).

[2] T. Takagahara, J. Lumin. 44, 347 (1989).

[3] L.C. Andreani, Solid State Commun. 77, 641 (1991).

[4] L. Schultheis, A. Honold, J. Kuhl, K. Köhler, C.W. Tu, Phys. Rev. B 34, 9027 (1986).

[5] E. Hanamura, Phys. Rev. B 38, 1228 (1988).

[6] N.T. Pelekanos, J. Ding, M. Hagerott, A.V. Nurmikko, H. Luo, N. Samarth, J. Furdyna, Phys. Rev. B 45, 6037 (1992).

[7] P.M. Young, E. Runge, M. Ziegler, H. Ehrenreich, Phys. Rev. B 49, 7424 (1996). 